\title{
CHIC (Charm in Heavy Ion Collisions): An experiment to measure charm production at the CERN SPS.
}

\section{Frédéric Fleuret*}

LLR, École polytechnique, CNRS-IN2P3, Palaiseau, France

\begin{abstract}
We propose an experiment to perform a systematic study of charm production in heavy ion collisions at SPS. Taking advantage of significant advances in electromagnetic calorimetry, the measurement of low energy photons from $\chi_{c}$ decays should now be achievable. Together with recent measurements made at RHIC and at LHC on $J / \psi$ and $\Upsilon$ production, such measurements will offer the necessary information to use quarkonia as a direct test of phase transition and lattice QCD calculations.

This new and dedicated experiment is designed to also study, under optimal conditions, Cold Nuclear Matter (CNM) effects in a larger rapidity range than previously explored by the NA50/NA60 experiments. This measurement of nuclear effects in absence of Quark Gluon Plasma formation will provide a clear and unambiguous reference for the study of Hot and Dense Matter (HDM) effects, a reference which is today needed to deduce an unambiguous interpretation of the results already obtained.
\end{abstract}

XXI International Workshop on Deep-Inelastic Scattering and Related Subject -DIS2013, 22-26 April 2013

Marseilles, France

*fleuret@in2p3.fr 


\section{Introduction}

Twenty five years ago, CERN pioneered the study of quarkonium production in heavy ion collisions at the SPS with the aim of characterizing the QGP phase transition and testing lattice QCD predictions. In 1997, the NA50 experiment observed an anomalous suppression of $J / \psi$ production in $\mathrm{Pb}-\mathrm{Pb}$ collisions [1]. Since then, these quarkonium studies have been extended to regimes of significantly higher energies as reached at BNL-RHIC and CERN-LHC. In addition with the results obtained at RHIC on $J / \psi$ and more recently at LHC on quarkonium states, hints of the theoretically expected sequential suppression [2] start to emerge.

Nevertheless, the experimental validation of such a scenario as well as the characterization of the phase transition require full control of the feed-down sequence. In particular, a precise measurement of quarkonium $1 \mathrm{P}$ states which significantly contribute to the yields of quarkonium states is mandatory. For charmonium, the measurement of $\chi_{c}$ suppression together with $J / \psi$ and $\psi^{\prime}$ is needed to prove the sequential suppression scenario. Such measurements should be performed under dedicated experimental conditions where the energy density is appropriate for the physics case.

Moreover, Cold Nuclear Matter effects which affect the extraction of Hot and Dense Matter effects must be thoroughly measured and well under control. Despite all the experimental results already obtained on $J / \psi$ production in p-A collisions, they are still not well understood, especially at small Bjorken $\mathrm{x}\left(x_{B}\right)$ and large Feynman $\mathrm{x}\left(x_{F}\right)$ where these effects are expected to be large.

\section{Charmonium production in A-A collisions}

$J / \psi$ and $\psi^{\prime}$ production studies in heavy ion collisions at SPS energies have been previously studied by the NA38, NA50 and NA60 experiments [1][3][4][5][6][7]. Figure 1, extracted from [6] shows, for various interacting systems, the ratio between the measured charmonium yields, normalized to Drell-Yan dimuons, and the corresponding expected "normal nuclear absorption", where the latter is computed from $\mathrm{p}$-A results. The quantity $L$, the length of nuclear matter seen by the $c \bar{c}$ pair, is calculated through the Glauber model formalism [8] of nucleus-nucleus collisions. The figure shows that the departure from ordinary nuclear absorption occurs at a lower value of $L$ for $\psi^{\prime}$ than for $J / \psi$.

The observed suppression patterns are compatible with the sequential suppression scenario where one expects the $\psi^{\prime}$ resonance to be screened by the QGP at a smaller $L$ (smaller energy density) than the $J / \psi$. In this scenario, the three charmonium states, $\psi^{\prime}, J / \psi$ and $\chi_{c}$, are suppressed at different threshold energy densities. Because of $\psi^{\prime}$ and $\chi_{c}$ feed-downs $(\sim 10 \%$ and $\sim 30 \%$ respectively), a sequential suppression should be observed when measuring the $J / \psi$ yield. Experimentally, because of the small $\psi^{\prime}$ contribution and its small production yield, it is very difficult to test the sequential screening by measuring $J / \psi$ and $\psi^{\prime}$ only. Checking whether the onset of $\chi_{c}$ suppression coincides with a sizeable suppression of $J / \psi$ would be a much more conclusive test.

An alternative scenario, not involving QGP, is the suppression of the resonances by their interaction with comoving hadrons, the so-called comovers [9]. In this case, the three charmonium states 


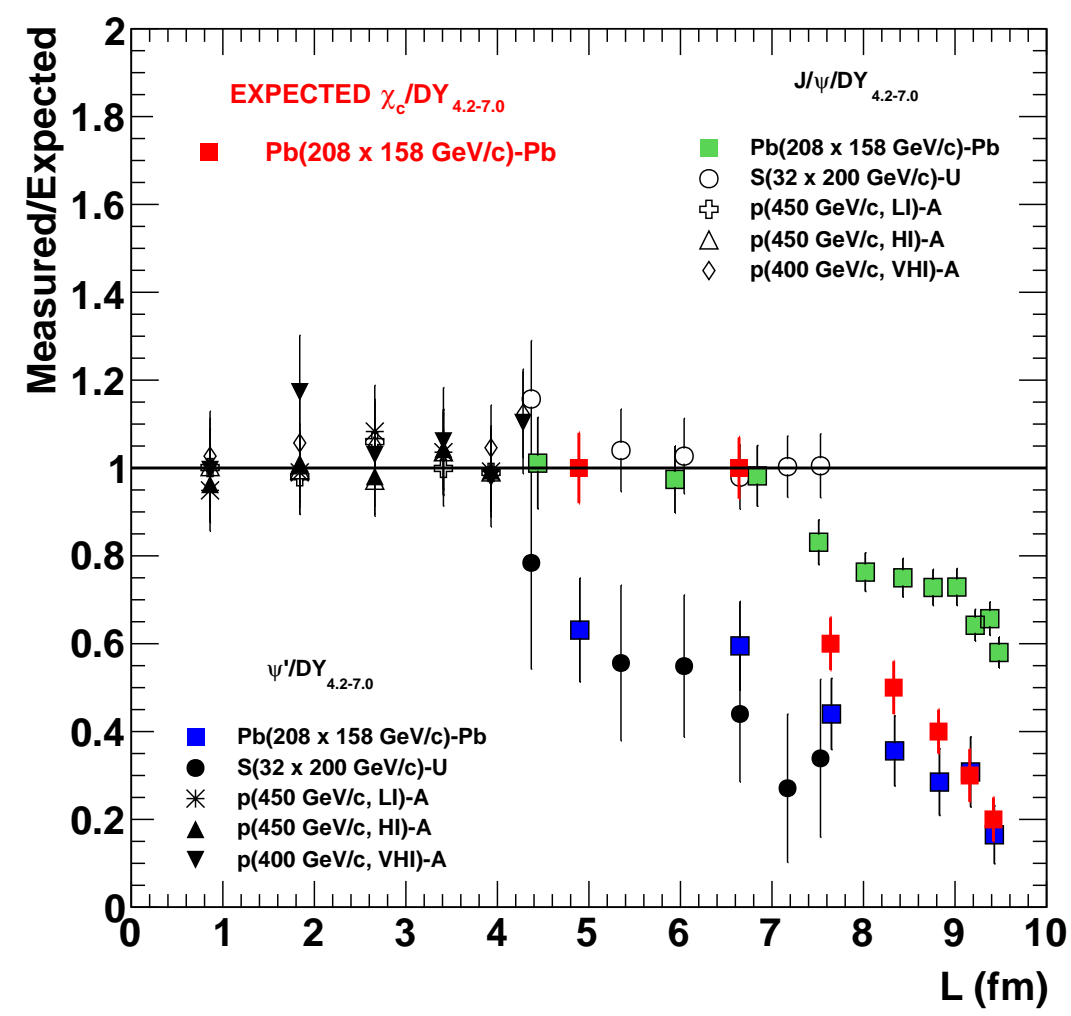

Figure 1: The ratio "measured over expected" for the relative yields $B_{\mu \mu}^{\prime} \sigma\left(\psi^{\prime}\right) / \sigma(D Y)$ and $B_{\mu \mu} \sigma(J / \psi) / \sigma(D Y)$, as a function of $L$. Figure taken from [6]. Red points show the expected precision obtained with $3000 \chi_{c}$ recorded during a typical 40-day $\mathrm{Pb}-\mathrm{Pb}$ run with CHIC (see section 4).

start to be suppressed at the same energy density but with a different slope. Therefore, the $\psi^{\prime}$ and $\chi_{c}$ feed-downs should only affect the slope of the inclusive $J / \psi$ suppression.

To test the phase transition and discriminate between the two scenarios, one must measure the three charmonium states. Because of its $\sim 30 \%$ feed-down contribution to the $J / \psi$ yield, the $\chi_{c}$ is a much better candidate than the $\psi^{\prime}$ to test the sequential suppression. In addition, the measurement of its suppression pattern should probe if comovers play an important role in the charmonium suppressions. Indeed, because of its smaller binding energy when compared to the $J / \psi$, larger when compared to the $\psi^{\prime}$, the $\chi_{c}$ suppression pattern should stand in between the $\psi^{\prime}$ and $J / \psi$ ones.

\section{Charmonium production in $\mathbf{p - A}$ collisions}

The study of charmonium production in proton induced collisions is crucial for a correct interpretation of the charmonium suppression patterns observed in heavy-ion collisions. It is, indeed, very important to establish a robust baseline with respect to which we can clearly identify new phenomena such as QGP. It is, in addition, crucial to understand the physics mechanisms underlying charmonium productions [10]. 
Several effects can be studied, such as the interaction of the evolving $c \bar{c}$ pair with the target nucleons leading to a break up of the charmonium states, parton shadowing (or antishadowing) in the target nucleus [11] which may suppress (or enhance) the probability of charmonium production, providing information on the $c \bar{c}$ pair production kinematics [12], saturation effects, parton energy loss [13].

Several experiments have studied $J / \psi$ and $\psi^{\prime}$ production in p-A collisions from $\sqrt{s_{N N}} \sim 20$ $\mathrm{GeV}$ to $\sqrt{s_{N N}}=200 \mathrm{GeV}$ (at LHC a p-Pb run at $\sqrt{s}=5 \mathrm{TeV}$ has been done in january 2013). However, mostly due to small statistics, little is known about $\chi_{c}$ production in p-A collisions (for a review of all the experimental data, see [14]). Moreover, the data collected by the NA60 experiment at $\sqrt{s_{N N}}=17.2 \mathrm{GeV}$ (158 GeV in the lab frame) to study $J / \psi$ and $\psi^{\prime}$ production in p-A collisions [15], cover a rapidity range too small to establish a robust and unambigous reference for the study of charmonium production in A-A collisions. Therefore, the achievement of a p-A program, covering a large rapidity range at both $\sqrt{s}=29.1 \mathrm{GeV}$ and $\sqrt{s}=17.2 \mathrm{GeV}$, to study $J / \psi$, $\psi^{\prime}$ and $\chi_{c}$ production on different targets would provide key information on the physics mechanism underlying charmonium production and needed baselines for the study of a phase transition in $\mathrm{Pb}-\mathrm{Pb}$ collisions.

\section{Experimental apparatus and expected performances}

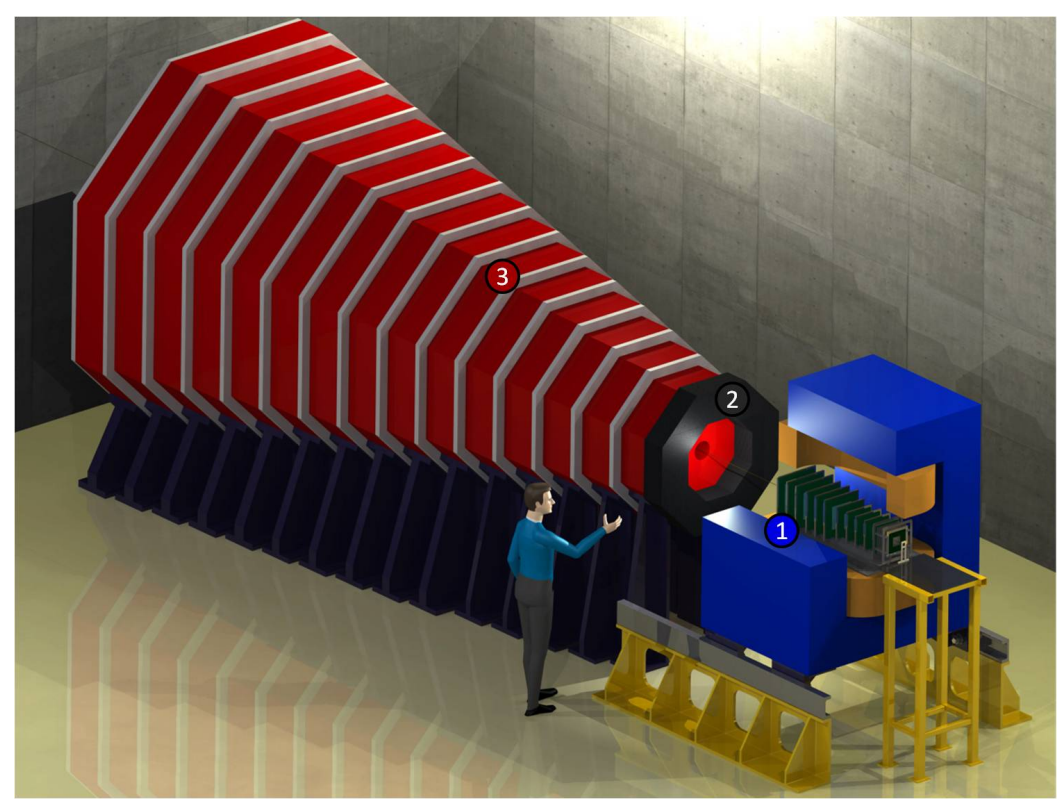

Figure 2: Experimental apparatus: 1) Vertex/tracking. 2) EMCal. 3) Absorber/trigger system.

The experiment proposed in this document will mainly consist of three detector components: a vertexing and tracking telescope, an electromagnetic calorimeter and a trigger system. The detector design is driven by two motivations:

- Since Hot and Dense Matter (HDM) effects, such as QGP, depend on the energy density, they are large at mid rapidity. This region has been investigated by all the experiments 
involved in quarkonium measurement, in heavy ion collisions. We adopt the same strategy, both because the HDM effects are larger and because previous measurements are available for comparison. Thus, the detector must be able to measure $\chi_{c}$ production in A-A collisions within the rapidity range $y_{C M S} \in[-0.5,0.5]$ in the center-of-mass of the collision.

- To improve the studies of Cold Nuclear Matter effects already made by the NA50/NA60 experiments, the spectrometer must be able to cover a larger rapidity range. With an appropriate design, we estimated that the rapidity coverage can reach $y_{C M S} \in[-0.5,2]$ in p-A collisions.

These two requirements lead to the detector design shown in Figure 2:

1. Vertexing and tracking system: We estimated that a similar detector than the NA60 pixel telescope extended over a one meter long dipolar field of $2.5 \mathrm{~T}$ would lead to momentum resolution of $1 \%$ giving a $J / \psi$ mass resolution on the order of $20 \mathrm{MeV} / \mathrm{c}^{2}$, five times better than what has been done before and sufficient to fully separate $J / \psi$ and $\psi^{\prime}$ states.

2. Electromagnetic calorimeter: Because of the very high multiplicity in A-A collisions, a very highly segmented calorimeter is mandatory in order to be able to isolate and measure each photon reaching the calorimeter. Such a detector is being developed by the CALICE collaboration [16]. We have estimated that, for a typical 5\% most central $\mathrm{Pb}-\mathrm{Pb}$ collision, such a calorimeter located at a distance of 205 centimeters from the vertex would permit to reach our goal. The energy resolution of such a detector would be better than $\Delta E / \sqrt{E} \sim 20 \%$ giving a $\chi_{c}$ mass resolution on the order of $45 \mathrm{MeV} / \mathrm{c}^{2}$.

3. Absorber/trigger system: When measuring $J / \psi \rightarrow \mu^{+} \mu^{-}$one has to deal with a huge background arising from $\pi^{+/-}$and $K^{+/-}$decays. In order to limit the trigger rate and the signal contamination, it is necessary to absorb these hadrons or their decay muons before they reach the trigger detectors. We estimated that a $4.5 \mathrm{~m}$ long Fe absorber would absorb most of the muons coming from pion decays leading to a trigger rate of $\sim 300 \mathrm{~Hz}$ for a $10^{7} \mathrm{~Pb}$ ions/sec beam delivered on a $0.4 \mathrm{~cm}$ thick $\mathrm{Pb}$ target.

Performances in $\mathrm{PbPb}$ collisions: For the $\chi_{c}$ study, we based our estimate on the same beam intensity as delivered to the NA50 experiment in [6], hence $5 \times 10^{7}$ ions per burst. Within our detector acceptance, we expect $\sim 180,000 \mathrm{~J} / \psi$ and $\sim 1300 \psi^{\prime}$ in a typical 40-day data taking period with a $10 \% \lambda_{I} \mathrm{~Pb}$ target. For the $\chi_{c}$, we expect, whether $\chi_{c}$ is suppressed as $\psi^{\prime}$ or $\chi_{c}$ is suppressed as $J / \psi$, between $\sim 3000$ and $\sim 7000 \chi_{c}$ respectively. Figure 1 shows the expected performances in the configuration " $\chi_{c}$ as $\psi^{\prime \prime}$ where red points are possible $\chi_{c}$ Measured/Expected ratio.

Performances in $\mathbf{p} A$ collisions: As reported by the NA50 experiment in [17], thanks to the high intensity proton beam, several hundred of thousands $J / \psi$ can be collected on a typical 1 week run on a given target. With the current SPS operation, delivering proton beam to the LHC several months per year, we expect to be able to collect a significantly larger amount of data.

\section{Conclusion}

In summary, we propose a new fixed target experiment at CERN-SPS in order to accurately study charmonia production in proton and heavy ion induced collisions. In heavy ion collisions, 
the measurement of $\chi_{c}$ together with $J / \psi$ and $\psi^{\prime}$ will provide crucial information on deconfined matter. In proton-nucleus reactions, the study with several targets of the three charmonium states in large $x_{B}$ and $x_{F}$ ranges will provide key information on Cold Nuclear Matter effects and a robust baseline with respect to which we can clearly identify specific phenomena occuring in heavy ion induced collisions.

Experimentally, we propose to adopt the same strategy as used for modern particle physics apparatus: we intend to measure muons before they reach the hadron absorber. For the photon

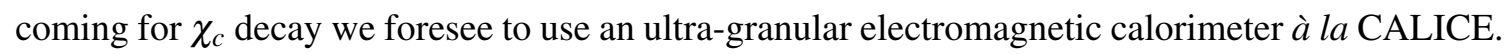
For the triggering system, an instrumented magnetized Fe absorber is envisionned.

With such an apparatus, we expect a mass resolution of $\sim 20 \mathrm{MeV} / \mathrm{c}^{2}\left(\sim 45 \mathrm{MeV} / \mathrm{c}^{2}\right)$ for the $J / \psi\left(\chi_{c}\right)$. For a typical 40-day run with $\mathrm{Pb}$ beam delivered to a $10 \%$ interaction length $\mathrm{Pb}$ target, we expect to collect more than $3000 \chi_{c}$.

Finally, even though it is not discussed in the present document, our experimental apparatus is very well suited to explore other important physics subjects such as open charm or low mass lepton pairs production in heavy ion collisions.

\section{References}

[1] NA50 Collaboration, Phys. Lett. B410, 337 (1997)

[2] F. Karsch, D. Kharzeev and H. Satz, Phys. Lett B637, 75 (2006)

[3] NA38 Collaboration, Phys. Lett. B466, 408 (1999); Phys. Lett. B444, 516 (1998)

[4] NA51 Collaboration, Phys. Lett. B438, 35 (1998)

[5] NA50 Collaboration, Eur. Phys. Jour. C48, 329 (2006); Eur. Phys. Jour. C39, 335 (2005)

[6] NA50 collaboration, Eur. Phys. Jour. C49, 559 (2007)

[7] NA60 Collaboration, Phys. Rev. Lett. 99, 132302 (2007)

[8] R.J. Glauber, Lectures in theoretical Physics, Ed. by W.E. Brittin and L.G. Dunham (Interscience, NY, 1959) vol. $1,315$.

[9] A. Capella et al., Nucl. Phys. A698, 583-586 (2002);

N. Armesto et al., Phys. Rev. C59, 395-404 (1999)

[10] Z. Conesa del Valle et al., Nucl. Phys. Proc. Suppl. 214, 3-36 (2011)

[11] D. de Florian, R. Sassot, Phys. Rev. D69, 074028 (2004) K. J. Eskola, H. Paukkunen, C. A. Salgado, JHEP 0904 (2009)

[12] E. G. Ferreiro et al., Phys. Lett. B680, 50 (2009)

[13] F. Arleo, S. Peigné, Phys. Rev. Lett. 109, 122301 (2012)

F. Arleo, S. Peigné and T. Sami, Phys. Rev. D 83, 114036 (2011)

[14] HERA-B Collaboration, Phys. Rev. D79, 012001 (2009)

[15] NA60 Collaboration, Phys. Lett. B706, 263-267 (2012)

[16] https://twiki.cern.ch/twiki//bin/view/CALICE

[17] NA50 Collaboration, Eur. Phys. Jour. C33, 31 (2004) 